\title{
A Comparative Study of Orientation Support Tools in Virtual Reality Environments with Virtual Teleportation
}

\author{
Matthias Kraus* \\ Hanna Schäfer" \\ University of Konstanz \\ University of Konstanz \\ Philipp Meschenmoser* \\ Daniel Schweitzer \\ University of Konstanz \\ University of Konstanz \\ Daniel A. Keim*
University of Konstanz \\ Michael Sedlmair ${ }^{\dagger}$ \\ Johannes Fuchs ${ }^{*}$ \\ University of Stuttgart University of Konstanz
}

\begin{abstract}
Movement-compensating interactions like teleportation are commonly deployed techniques in virtual reality environments. Although practical, they tend to cause disorientation while navigating. Previous studies show the effectiveness of orientation-supporting tools, such as trails, in reducing such disorientation and reveal different strengths and weaknesses of individual tools. However, to date, there is a lack of a systematic comparison of those tools when teleportation is used as a movement-compensating technique, in particular under consideration of different tasks. In this paper, we compare the effects of three orientation-supporting tools, namely minimap, trail, and heatmap. We conducted a quantitative user study with 48 participants to investigate the accuracy and efficiency when executing four exploration and search tasks. As dependent variables, task performance, completion time, space coverage, amount of revisiting, retracing time, and memorability were measured. Overall, our results indicate that orientation-supporting tools improve task completion times and revisiting behavior. The trail and heatmap tools were particularly useful for speed-focused tasks, minimal revisiting, and space coverage. The minimap increased memorability and especially supported retracing tasks. These results suggest that virtual reality systems should provide orientation aid tailored to the specific tasks of the users.
\end{abstract}

Index Terms: Human-centered computing-Human computer interaction $(\mathrm{HCI})$ - Interaction paradigms-Virtual reality

\section{INTRODUCTION}

Virtual reality is currently a popular trend in several areas: a developing private market in the computer games sector, in the industry for business solutions, but also in research - for example, in the fields of human-computer interaction and data visualization. However, while the virtual environment can be of any size, the 'play area' i.e., the physical space available in the real world - is usually much smaller, leading to a discrepancy between the two worlds. Naturally. one would walk around for spatial navigation, leading to improved orientation abilities due to self-motion [27,42]. However, often the available physical space is limited, confining this interaction in virtual reality. Razzaque et al. [41] presented 'redirected walking' as a technique for enlarging the virtually accessible space by tricking the orientation and perception of users. In their approach, the virtual environment is rotated, unnoticed by the user, detaching the spatial mapping of the real world from the virtual world. Thus, the user's physical movement can be limited to a small area, while the virtual range is (theoretically) unlimited. However, to keep physical direction changes unnoticeable to the user, it is necessary that the

"e-mail: \{firstname.lastname\}@uni.kn

†e-mail: michael.sedlmair@ visus.uni-stuttgart.de user walks in wide arches, which makes the technique unsuitable in confined physical space (e.g., $3 \times 3 \mathrm{~m}$ ).

Another way to alleviate the problem of limited physical space is to intercept the physical input (i.e., walking) and simulate it in the virtual environment. So-called VR treadmills [36] are large installations, on which a VR user can walk on the spot, covering virtual space while staying in the same physical location and still using natural interaction as a means of navigation. However, the approach has disadvantages: Besides a bulky setup and expensive hardware, the approaches are not yet fully developed and do not perfectly mimic real interactions [45].

Indirect spatial navigation via buttons or joysticks, typically used in computer games, is an alternative way of overcoming the differences between the virtual and the real world. However, this type of movement fosters an asynchronous movement between body and perspective, which is unfamiliar to our brain and can, thus, lead to physical discomfort - often referred to as simulator or motion sickness $[23,30]$.

Another alternative is virtual 'teleportation', which circumvents the aforementioned problems associated with asynchronous movement. The user points to a specific location within sight and triggers the movement with a controller or similar. The user's location is then abruptly set to the selected position. Initial research suggests that this might not be the ultimate solution either, as it fosters a loss of orientation, since the point of view is often changed without physical transition of the user $[4,50]$. Various orientation aids can be deployed to counteract such losses of orientation, such as trails or minimaps.

In this paper, we seek to better understand how well different visual orientation-supporting tools compensate this disorientation problem by evaluating and comparing three typically used tools collected from literature. Orientation-supporting tools are means helping us to maintain our orientation in (VR) environments, even when it is made difficult by unfamiliar movement techniques such as virtual teleportation. Navigational tools can be considered as a subgroup of orientation-supporting tools. Navigational tools require the destination to be known so that the system can help the user to find a way to this target location. We focus on environments where the physical space is limited, and teleportation is deployed as a movement-compensating method. We selected three techniques for evaluation. Two of them are frequently used for facilitating orientation: a minimap and trailblazing. As a third technique, we investigated the performance of real-time heatmaps to support orientation. In a user study with 48 participants, we compared these three approaches to each other and to a baseline scenario without any orientation-supporting tools.

The main contributions of our research are (i) the results of a between-subjects user study that assesses and compares three passive and stand-alone orientation-supporting tools in virtual reality environments (VREs) with limited physical space that use teleportation for spatial movement, and (ii) a set of guidelines that propose types of tools for different scenarios and tasks. 


\section{BACKGROUND}

In the following, we provide definitions of prominently used terms, as well as an overview of various application scenarios in which navigation and orientation-supporting tools play a role. Additionally, we give an overview of existing techniques and categorize them according to distinguishing properties.

\section{1 (Digital) Wayfinding and Orientation}

Darken and Peterson [10] define navigation as a combination of wayfinding and motion, with wayfinding being the cognitive elemen and motion the motoric element. Further, spatial cognition is "concerned with the acquisition, organization, utilization and revision of knowledge about spatial environments" [17]. Spatial orientation is associated with spatial cognition and describes the ability to orien oneself relative to specified positions on a cognitive map. A highe degree of orientation increases the performance in wayfinding and navigation tasks $[17,32]$. Orientation is a complex construct that can affect many aspects that rely on one's abilities to grasp and understand the spatial structure of an environment and its spatia relationships. In the current evaluation, we make use of three indicators for improved orientation: the ability to gain and maintain an overview of the environment, the ability to return to previously visited places, and the ability to maintain an overview of all previously visited places.

While it is apparent that orientation is important in navigation scenarios where the main task is to find an (optimal) path from one location to another, it is also important in other scenarios withou concrete navigational tasks. For example, in visual exploration scenarios in which the user navigates through a large data space, it is important that analysts can maintain their orientation. Examples are the exploration of multidimensional data visualized as scatterplots in immersive environments [15] or the exploration of digital model of cities [51], buildings [40] or crime scenes [25]

Previous work has already investigated in detail how navigation can be supported by providing additional visual aid. Thereby, the research is based on real-world applications where technology was developed and evaluated to guide users through real environments. A classic example is the navigation system in a car. The location of the car is shown on a map and a route is highlighted that suggests where the driver should go. Besides, there is a large number of research projects dealing with navigational support in digital environments i.e., screen-based applications where users are guided from one location to another $[11,14,43]$. The prevalent examples are gaming and simulation applications where a user navigates in a virtual real world simulation.

\subsection{Improving Orientation in VR}

Another branch of research deals with orientation and how it can be improved in virtual reality environments. In this area the ultimate goal is to develop techniques that support users in improving their cognitive map of an environment.

Virtual reality environments are frequently used to study basic human navigation and orientation capabilities in laboratory setup $[33,34,49]$. For instance, Moffat et al. [34] examined the influence of age on participants' ability to navigate in an abstract labyrinthine environment and found an impairment of orientation that correlated with age. Similarly, Maguire et al. [33] investigated the influence of age and gender on orientation capabilities and found indications for interactions between the two properties and orientation, for example, lower orientation skills with increasing age.

Virtual reality is a relatively new trend that is constantly expanding into new applications. Although the environments explored are digital, and in this respect similar to screen-based digital environments, due to immersion, navigation in VR is more similar to navigation in the real world. Therefore, various techniques for navigation and orientation support were transferred from screenbased applications to VR and partially evaluated, for example, trails, compasses, and minimaps $[1,11,12]$.

Apart from that, techniques were developed and evaluated that are tailored to stereoscopic vision and 3D space provided in virtua reality environments, such as 3D minimaps [6], 3D radars [5], edge radars [19], or fisheye views [44]. Kotlarek et al. [28] compared landmarks, 3D minimaps and waypoint navigation with regard to their ability of improving spatial orientation and identified the 3D minimap as the most efficient aid in their comparison. Besides providing explicit visual tools for orientation improvements, several tudies considered more indirect ways to improve orientation. For example, Müller et al. [35] describe how collaborative VR environments can be configured in order to foster orientation. They found that shared virtual artifacts increased orientation in remotely co-located virtual environments for collaborative tasks.

Riecke and Schulte-Pelkum [42] identified self-motion as a crucial element for orientation and set out how the illusion of selfmotion (vection) could be used in virtual environments to enhance orientation. This is in particular important for VREs with limited physical space as they commonly rely on alternative locomotion techniques to walking. The impact of using movement compensating techniques, such as teleportation [4] or controller-based move ment [50] on orientation has been investigated in previous studies. Bhandari et al. [3] presented 'Dash', an alternative technique to teleportation in which a user is slowly transitioned to a selected location and compared it to its original alternative. They found that provided optical flow cues in their new technique increased orientation. Similarly, Langbehn et al. [30] compared redirected walking, joystick navigation and teleportation and found an advantage of redirected walking compared to the other two approaches, as it helps the user to unconsciously acquire spatial knowledge of the virtual environment.

However, in many VR applications the use of standard virtua teleportation still predominates. Therefore, we complement to the line of research of finding suitable tools to counter orientation loss induced by virtual teleportation by comparing three well-established, visual orientation-supporting tools in virtual reality environments.

\subsection{Types of Visual Orientation Support}

We categorize existing techniques of visual orientation-supporting tools according to three characteristics: environment dependency, target dependency, and action dependency.

Environment dependency: Environment-dependent tools are tools where the environment has to be attuned to the respective task. An example of an environment-dependent orientation-supporting technique is sectioning $[13,37]$. In this approach, the environment is divided into visually distinct segments. For example, in the virtual model of a building each level could have a carpet in a different color. The approach of landmarking $[31,37,46]$, in which visually distinguishable landmarks (e.g., objects as salient cues) are placed in the virtual environment to serve as orientation anchors, is also environment-dependent. With both approaches, the environment has to be adapted beforehand. Environment-independent or stand-alone approaches, on the other hand, are applicable without manipulating the environment itself. Examples are minimaps or static directional cues, such as a pointer to the north or a virtual sun [10].

Target dependency: Any navigational support that guides the user to a system known location is target-dependent. Conventional navigation guides, such as minimaps with a given target location or even a suggested path, are examples of this category [10], as well as radars or arrows indicating target locations $[5,7]$. Target-independent approaches are all approaches that do not take the target location into account, such as the breadcrumb or footprint technique, which depicts a person's travel history on the ground $[18,43]$.

Action dependency: Action-dependent approaches require active user interaction. The predominant example is interactive landmark- 
ing [8]. With this approach, the user can place different objects as spatial cues in the virtual environment to improve his or her orientation. Any other passive tool that does not require active user interaction is categorized as action-independent.

In our work, we focus on environment-independent, targetindependent, and action-independent orientation-supporting tools in virtual reality environments, as they are the most versatile aids for exploration tasks.

\section{Assessing ORIENTATION-SUPPORTINg TOOLS IN VR}

In this paper, we assess and compare three different orientationaiding tools in VREs. We focus on VREs with limited physical space, which therefore use teleportation as a means of spatial movement. To test our assumptions, we conducted a quantitative user study. The study prototype was developed with the gaming engine Unity $3 \mathrm{D}$ [48]. In the following, we will first present the rationale behind the conducted evaluation and justify the selection of three orientationsupporting tools before continuing with a detailed description of the conducted user study.

\subsection{Research Objectives \& Study Design}

Teleportation is a technique frequently used in VREs to overcome limited physical space while avoiding the deployment of expensive hardware or hazarding the risk of increased levels of simulator sickness caused by asynchronous body and viewpoint movements. Bowman et al. [4] found that the use of teleportation for spatial movement in virtual reality environments has an impact on spatial orientation and suggest the deployment of alternative ways of relocation instead of teleportation. Our goal is to investigate possible solutions for the loss of orientation while maintaining the popular technique of teleportation for movement. There are several techniques that are developed to support a user's orientation. Our key research questions are: Do common orientation-supporting techniques indeed help users with orientation? Which technique is best suited to maintain a user's orientation in a complex environment that promotes disorientation?

The conducted study comprised only one experimental factor: the provision of a specific orientation-supporting tool. Since our goal was to compare three different techniques with each other and with the provision of no tool at all, trials were carried out in four conditions. The study design was a between-subjects design and included 48 participants in total. Thus, the sample was divided into four groups of 12 participants, each group using one of the three orientation-supporting tools or none at all.

We used mazes of different sizes as abstract environments to promote disorientation. However, to test the applicability of our findings, we also used a session in which participants performed search task in a realistic environment. To investigate the orientation of users, we used different exploration and search tasks that allowed us to quantify orientation.

We focused on the comparison of the orientation-supporting tools in their basic form, without considering any combinations of them. Based on these initial results, future research should evaluate the pairings of approaches as well. As far as the selection of examined techniques is concerned, we selected well-functioning and optimized techniques from other domains or evaluations that meet our requirements.

\subsection{Conditions: Orientation-Supporting Tools}

We evaluated four orientation-supporting tools in our study.

\subsubsection{None}

In this baseline condition, participants completed the same trials as the other participants but were not equipped with any orientationsupporting tool. This condition was introduced to check whether the supportive tools have any positive benefit for user orientation and to serve as a baseline for comparison.

\subsubsection{Minimap}

Minimaps are frequently used navigational and orientationsupporting aids. In the case of our study, we wanted to focus on orientation-supporting aids. Hence we only used scenarios where the target location was unknown. Following the design principles of Darken and Peterson [10], we used a north-up map, which is optimized for geocentric tasks like explorations or naive searches (see Figure 1, left). For egocentric tasks, such as navigating to a known location, forward-up maps performed better in previous evaluations [12]. To ensure optimal performance of the map, we added a user icon that always indicates the user's position and orientation on the map, as suggested by Darken and Peterson [10]

In the study, the minimap was attached to the user's left controller and could be moved around as desired. An orthographic camera was centered above the respective scenario, so that the user-accessible bounds were aligned with the bounds of the map. This means that the entire environment was continuously visible on the minimap. The result can be compared with a map of the ground plan of the scenario. Task-based markers were not depicted in the minimap.

\subsubsection{Trail}

As shown in Figure 1 (center), the orientation-supporting tool Trail draws a trail behind the user while he or she moves in the virtual space. Arrows on the trail indicate the direction in which the path was taken. Based on Darken and Sibert [13], we decided against a breadcrumb approach and instead used a continuous line with directional cues - a directed trail. Continuity makes the trail more robust against overlaps and crossings, as opposed to, for example, footprints [18]. Directional cues, such as textures on the trail that indicate the direction in which the path was taken, increase the user's ability to keep track of the path, and reconstruct the history of movements. Darken and Peterson [10] express concern about the use of trails as they clutter the space after long sessions, but at the same time point out that the alternative of fading trails can lead to confusion because the aid no longer provides binary information about whether or not a particular place has been visited before. As we designed our sessions to be short (about 5 minutes) and used environments with a small areas (maximum of $100 \mathrm{~m}^{2}$ ), we retained all trails from the beginning to the end of a session. Furthermore, we abstained from visually encoding the recency of a trail, for example, by color, as suggested by Ruddle et al. [43], since this would lead to non-persistent visualizations that change color over time, which in turn could lead to a loss of orientation.

Regarding the technical implementation, we used a textured line renderer, that is extended by waypoints as the user moves. We set a threshold of $20 \mathrm{~cm}$ to the latest point so that no new waypoints are created unless the user moves significantly. To smooth the corners of the displayed line, we implemented an interpolation function that makes the displayed trails more visually appealing. Normals of the lines are set to the normals of the ground plane. Finally, we set a threshold of waypoints for the line renderer. Once the threshold is reached, the line is converted to a mesh, and the line renderer is reset to improve performance.

\subsubsection{Heatmap}

The fourth scenario was inspired by applications that use heatmaps in post-processing steps to analyze user behavior. Heatmaps were used, for example, in gaze analysis procedures to visualize the eye movements of users $[16,47]$, and in sport analysis scenarios to analyze the movement of players in a soccer game $[2,38]$. We attempted to transfer the benefits found for such applications by using them as real-time updating heatmaps for orientation support. Unlike trails, heatmaps do not encode the direction from which a 

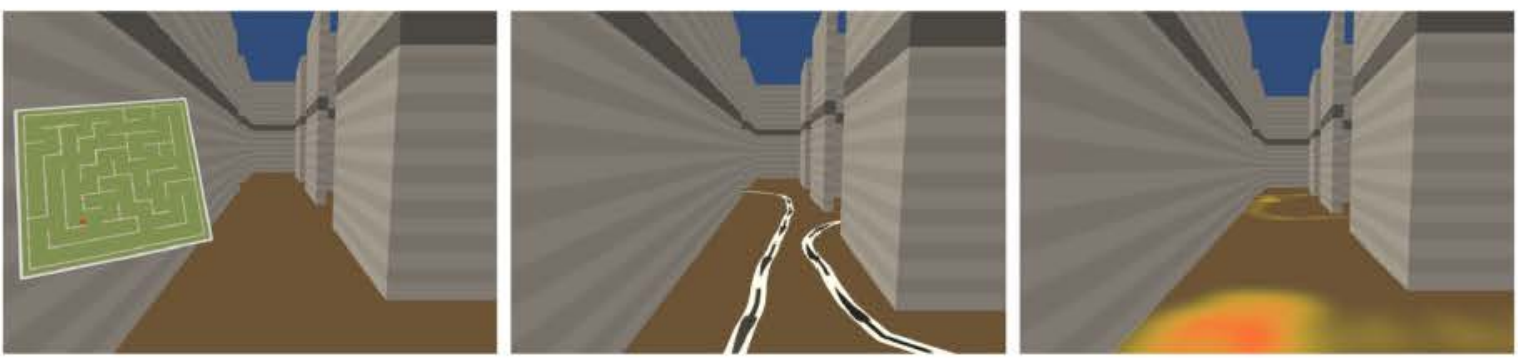

Figure 1: Provided orientation-supporting tools. Minimap (left): Attached to the left controller, a north-up minimap provides the user with an overview. The position and orientation of the user is depicted by a small icon on the map. Trail (center): As the user moves through the space, a trail is created highlighting the path taken. Heatmap (right): A heatmap on the floor depicts the history of user locations.

place was visited, but only the frequency with which that place was visited. This reduces the complexity of the presented visualization at the price of information loss. To implement the technique, the total area was divided into $10 \times 10 \mathrm{~cm}$ tiles on a $n \times m$ grid. Each tile served as a counter and monitored how long the player was standing on it. Every 0.5 seconds, the counter of the tile beneath the current player position was increased by one. To generate the heatmap, a $n \times m$ matrix was created, consisting of all counters. We then linearly normalized all non-zero values to a range between 0.1 and 1 . This normalization prevents spots in the heatmap from disappearing due to very high counts at certain positions. As a next step, we created a $n \times m$ texture from the matrix by assigning a color to each value based on our color gradient. As a color gradient, we deployed a linear color transition from fully transparent to yellow to red. Finally, we applied a Gaussian blur function [20] to the texture for smoothing. This Gaussian filter is a $N \times N$-tap convolution filter that weights all pixels in its catchment area based on the Gaussian distribution function. To visualize the heatmap, a texture plane was placed $1 \mathrm{~mm}$ above the floor. The texture of this plane was updated every two seconds (see Figure 1, right).

\subsection{Hypotheses}

Based on observations from a pilot study $(N=4$, same study prototype) and findings from relevant literature, we derived the following hypotheses. The evaluated scenarios were tailored to our hypotheses, focusing on a specific task type (exploration, naïve search, and informed search) and related task objectives (completeness of exploration, overview, and retrace abilities).

H1 We expect improved orientation when orientation-supporting tools are available. In general, we expect that participants are more efficient and more effective in exploration, naïve search and informed search tasks with all given tools, as previous research has shown a benefit of such tools in screen-based navigation applications [13]. This should be reflected, among other things, in lower task completion times, higher task accuracy for exploration tasks, and lower navigation times for informed search tasks.

H2 We expect that the orientation-supporting tool Heatmap increases the effectiveness in open exploration tasks. The heatmap indicates previously visited areas and thus implicitly points to areas that were not visited before. In contrast to the Trail visualization, it does not create clutter but provides a higher abstraction of the movement history. This should be reflected in a higher task accuracy for exploration tasks. Participants with a Heatmap are expected to leave less space unexplored and thus find more targets.

H3 We expect that the orientation-supporting tool Minimap offers the best overview in naïve search tasks. The Minimap provides a direct top-down view of the environment, revealing the structure of the environment and the user's position [13], helping the use to quickly gain and maintain an overview of the scenario to be explored. Therefore, we expect participants with a Minimap to be able to find a certain set of targets faster than participants without or with another tool when performing a naïve search task. In addition. we expect that participants are able to recall the structure of the environment better if they are provided with a Minimap.

H4 We expect that the orientation-supporting tool Trail increases tracing performance in informed search tasks - i.e., the ability of a user to navigate back to a known previously visited location. Ruddle et al. [43] demonstrated that this technique can be effective for subsequent search tasks. We, therefore, expect participants with the orientation-supporting tool Trail to find their way back to known locations fastest.

\subsection{Procedure}

Participants were invited to the laboratory for individual sessions. Prior to participation, all participants gave written informed consent. The experiment was divided into three parts. In the first part, participants filled in two standardized questionnaires assessing their mental rotation abilities and basic orientation skills: the Mental Rotation Test (MRT) by Peters et al. [39] and the Perspective Taking and Spatial Orientation test (PTSO) by Hegarty and Waller [22]. Such spatial ability tests are recommended by Darken and Peterson [10] because they make it possible to relate analysis results to participants spatial abilities. In the second part (main experiment), participants first completed a training procedure in VR in which all available interaction techniques were introduced (how to open markers, how to teleport). Subsequently, they performed four trials and answered questionnaires after each trial. Before each trial, participants took a short break and completed a calibration and instruction step in which they positioned themselves in the starting position (center of physical room) and read the instructions for the following trial. We provide a video, in which the four trials, as well as all conditions are graphically presented ${ }^{1}$. After the four trials were completed, the third part began, consisting of a customized questionnaire, a demographic questionnaire, and a semi-structured interview. Finally, participants were thanked and compensated for participating.

\subsection{Interaction Capabilities}

Regardless of the trial, participants could walk in the physical space available $(2.5 \times 2.5 \mathrm{~m})$. Additionally, participants could press and hold a button on the controller to select a location to which they were teleported after releasing the button. As a second interaction, participants could interact with markers by pointing at them and

\footnotetext{
${ }^{1}$ https://youtu.be/rnPvBTJQeh0
} 
pressing a button on the controller. This interaction was only possible from a distance of $0.5 \mathrm{~m}$. Once a marker was triggered ('opened'), it changed its appearance.

\subsection{Tasks \& Trials}

Darken and Sibert [13] distinguish between three types of tasks: exploration, naïve search, and informed search. We focused on the first two types, as we strove to investigate the performance of orientation-supporting tasks where the target location is not known to the system or the user. However, in order to also investigate user performance in informed search tasks, we constructed a naïve search task in which the user has to navigate back (retrace) to previously found targets, resulting in an informed search sub-task. Throughou the study, participants were asked to collect markers in four trials. However, the main objective of the specific task varied from trial to trial, defining its task type. The order of the trials was the same for all participants as we did not conduct cross-comparisons between task and strove to maintain the same learning effect for each participant in each task. In the following, we will elaborate on the descriptions of each trial.

Trial 1: Maze Exploration The main objective of exploration tasks is to explore as much of the environment as possible without leaving areas unexplored. Trial $1 \mathrm{can}$ be seen as an exploration task, as participants were asked to explore the respective scenario without knowing how many markers there are and when they are finished. Participants were instructed to explore the entire scenario carefully, as thoroughly as possible, and to collect all available markers. In the first trial participants were located in a $15 \times 15 \mathrm{~m}$ maze (see Figure 2, top left). Four markers were distributed in the maze. Participants were instructed to explore the maze and visit every corner of the maze. Moreover, they were instructed to collect al markers in the maze and to return to the starting position (blue circle on the floor) as soon as they are confident they have explored every inch of the maze. After this trial, participants filled in the NASA Task Load Index (TLX) questionnaire [21].

Trial 2: Naĩve Search Task The main objective in naïve search tasks is to find a set of targets efficiently. Trial 2 can be seen as a naïve search task as participants were asked to search for a certain number of markers. Participants were instructed to collect all four markers as fast as possible and return to the starting position.

The second trial took place in the same maze as the first trial. How ever, the four markers were placed in other locations. Participants were made aware of both circumstances. We used the same maze to exploit learning effects and to measure whether certain orientationsupporting tools enhance participants' mental map of the environment and thus facilitate the task. After this trial, participants again completed the TLX and a memorization questionnaire, assessing participants' ability to remember the structure of the maze

Trial 3: Informed Search Task The main objective in informed search tasks is to efficiently navigate to known locations. In trial 3 , participants were asked to collect markers in a specific order and return to the starting position. As soon as a marker was 'opened', i revealed a certain number $(1,2$, or 3$)$. We manipulated the numbers on the markers so that participants always found the third marker first, then the second, and then the first marker. So when they finally got to the first maker to collect, they knew the position of the other two markers. In this way, we were able to construct an informed search task that starts with a naïve search task where neither the system nor the user knows the targets in advance.

In the third trial, participants were located in a smaller, $10.5 \times 10.5$ $\mathrm{m}$ maze (see Figure 2, bottom left). Three markers with numbers ( 1 2 , and 3 ) were distributed in the maze. After this trial, participants filled in the TLX, a memorization assessment questionnaire, and the simulator sickness questionnaire (SSQ) by Kennedy et al. [26]
Trial 4: Real-World Inspired Exploration In the last trial, we used a more realistic scenario (see Figure 3 ) in which participants performed a similar exploration task as in trial 1. In the approximately $1,000 \mathrm{~m}^{2}$ large walkable space, ten $1 \mathrm{~cm}$ large diamonds were distributed. Participants were instructed to explore the environment for five minutes and collect all diamonds. After this last trial, participants again completed the TLX questionnaire.

\subsection{Scenarios / Virtual Environments}

For our study, we created two types of VREs: abstract and realistic.

\subsubsection{Abstract Scenarios: Mazes of Trials 1-3}

Darken and Sibert [13] found that people automatically take advantage of environmental cues and partition spaces for better orientation. We decided to minimize this benefit of natural-looking environments by designing the environments for the first three trials as monotonous and uniform as possible. In addition, we used mazes as they naturally promote disorientation, thus increasing the potential impact of orientation-supporting tools.

Figure 2 depicts the ground plans of both mazes used (left) and a screenshot of the first-person perspective of a participant in the maze (right). The blue dots in the ground plans represent participants starting positions. For the first two trials, a larger maze with a dimension of $15 \times 15 \mathrm{~m}$ was used. For the third trial, the dimension of the maze was $10.5 \times 10.5 \mathrm{~m}$. We used the same mazes for all participants to be able to compare their performance. The maze was not changed from the first to the second trial in order to exploit learning effects and to familiarize participants with the maze in the exploration task (trial 1) before they continued with the naïve search task of trial 2.

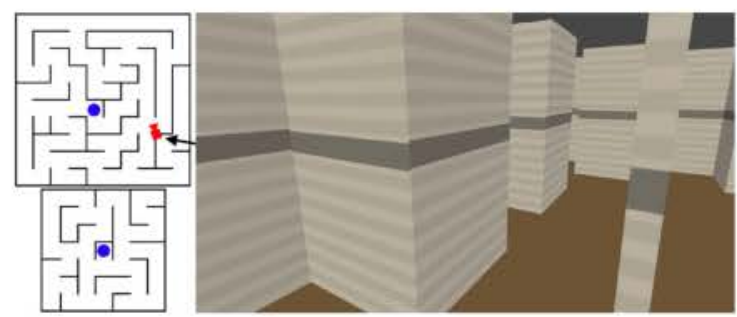

Figure 2: Mazes used in trials 1-3. Left: ground plans of mazes for trial 1 and 2 (top) and trial 3 (bottom). Right: sample image from the perspective of a participant standing in a maze.

The two mazes used were created randomly using a depth-first search algorithm. First, the ground plan of the maze was divided into $1.5 \times 1.5 \mathrm{~m}$ cells, and each cell was framed with four walls. One cell was randomly selected and marked as visited. For each cell currently visited, a list of neighbors was retrieved and processed in random order. For each neighbor not yet visited, the walls between the two were removed, and the same function was recursively called for this cell. We then removed various walls manually to retrieve unconnected walls. The sizes of the mazes used were obtained from pilot studies in which we measured the time participants needed to complete the given tasks in the respective mazes. We chose the mazes in which the average participant required 5 minutes to solve the task. Since the task in the third trial was more extensive compared to the first two trials, we decided to use a smaller maze for this trial. The texture of each maze was uniformly striped to enhance depth perception and the local spatial structure recognition of the maze, as participants in the pilot studies had difficulties in recognizing the corners of a monochrome maze. 


\subsubsection{Realistic Scenario: Industrial Environment of Trial 4}

Since mazes are rather unusual environments, we extended our study to include a more realistic use case that is inspired by a real-world scenario. Therefore, we created a fourth trial in which participants should explore a more realistic environment. For this, we used a 3D map of a fictional industrial environment. As a base environment. we adopted a map by Dimitrii Kutsenko [29] from the Unity3D asset store and adapted it slightly to fit our needs. Figure 3 depicts a top view of the scene (left) as well as a screenshot from the first-person perspective of a participant in VR (right). The red area marked in the left image illustrates the area that was accessible for the participant. The blue spot in the right image represents the starting position of the scene. The approximately $1.400 \mathrm{~m}^{2}$ comprise obstacles, such as pallet stacks, barrels, silos, and even houses, resulting in a total accessible area of about $1,000 \mathrm{~m}^{2}$.

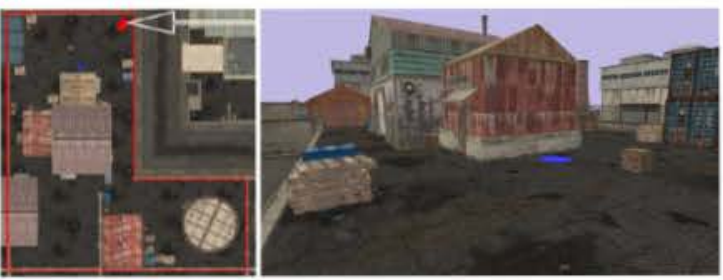

Figure 3: Scenario used for the real-world inspired exploration task (trial 4). Left: top view of the map. The red boundary indicates the area in which participants could move. Blue dots indicate participants starting position. Right: one image from the participants' perspective. The location from where this image was taken is marked as a small red camera in the left image.

\subsubsection{Markers}

Trials 1 to 3 were designed with large markers (Figure 4, left). The marker consists of a $35 \mathrm{~cm}$ cube with a $25 \mathrm{~cm}$ long diamond on top. Before interacting with the marker, the box was black with a red question mark, and the diamond was grey. Once opened by a user. the appearance changed. As the order of markers was irrelevant for trials 1 and 2, the diamond turned green and the box blue (see Figure 4, left). For the third trial, the order in which the markers had to be opened was relevant. Therefore, the box showed the identifier of the opened marker, and the diamond indicated whether the opened marker had to be opened next (green texture) or not (red texture), as depicted in Figure 4 (center). If the marker was not the right one, it returned to its original appearance after five seconds.

In the fourth trial, we used different and much smaller markers as the large ones were far too easy to recognize in the plain environment (see Figure 4, right). The markers used were $9 \mathrm{~mm}$ long diamonds of grey color. When opened, the markers were enlarged to a length of $5 \mathrm{~cm}$ and changed their color to green. Before the start of the trial, participants were made familiar with the appearance of the diamonds to be sought.

\subsection{Apparatus}

The experiment took place in a laboratory at the University of Konstanz. In addition to the participant, a study supervisor was present at each session. The physical space in which participants could move was an area of $2.5 \times 2.5 \mathrm{~m}$. Participants were equipped with an HTC Vive Pro [24] and two Vive controllers. To ensure maximum mobility, we used a wireless adapter for the Vive HMD.

\subsection{Sample}

A sample of $N=48$ participants ( 33 female, 15 male; aged between 18 and 43 years) was recruited using the study pool of the University
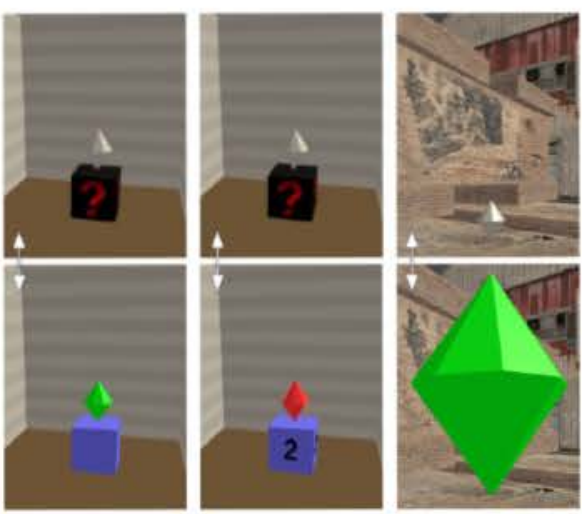

Figure 4: Used markers in the search and exploration tasks. Left: large markers of trials 1 and 2. Center: large markers with numbers of trial 3. Right: tiny markers of trial 4 ( $9 \mathrm{~mm}$ before opening, $5 \mathrm{~cm}$ after opening). The top image of each pair of images depicts the marker before opening, and the bottom one represents the marker after opened by a participant.

of Konstanz. Participants were sequentially assigned to one of the conditions depending on their gender to counteract possible gender differences in spatial orientation [9]. Median ages per condition were as follows: None $(M d n=23.5)$, Minimap $(M d n=24)$, Trail $(M d n=25)$, Heatmap $(M d n=23)$. Most participants did not have much experience with VR $(M=1.40, S D=0.22)$ and computer gaming $(M=2.15, S D=0.32)$. Means represent experience ratings of users on a scale from very few $=1$ to very much $=5$.

\subsection{Results}

We report significant results of our quantitative analysis as well as qualitative feedback from the final interview and the customized questionnaire. Statistical analysis was performed using IBM SPSS Statistics (version 25). All tests are based on a 0.05 significance level. Each dependent variable was first tested for normal distribution by the Kolmogorov-Smirnov test. We computed a one-way independent ANOVA for normally distributed data in combination with a TukeyHSD test as a post-hoc test. For non-normally distributed data, we used its non-parametric counterpart, the Kruskal-Wallis test, and a Mann-Whitney test as post-hoc test. Graphs display mean values, with error bars indicating standard errors of the mean. Asterisks indicate significant differences $(* p \leq 0.05, * * p \leq 0.01$, *ak* $p \leq$ 0.001 ).

Before the trials started, participants' basic mental rotation and orientation abilities were assessed with two standardized tests (MRT [39] and PTSO [22]). Statistical analysis did not reveal significant differences between groups, i.e., participants in the four conditions did not differ in their mental rotation or orientation skills (MRT: $H(3)=2.4, p=.494$; PTSO: $F(3,44)=1.49, p=.230$ ).

\subsubsection{Task Completion Time}

Task completion time was measured as the time difference between the start of the trial and the time of return to the starting position after completing the task. Since the time in trial 4 was limited to five minutes, we consider the task completion times only for trials one to three. For the maze exploration (trial 1), we corrected task completion times to the interval from the start of the trial to the last marker found as it was an open exploration task without a designated number of targets. Data for all three tasks considered were normally distributed. 


\section{Task Completion Times}
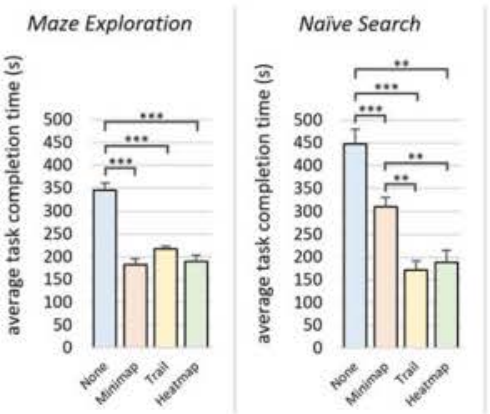

Informed Search

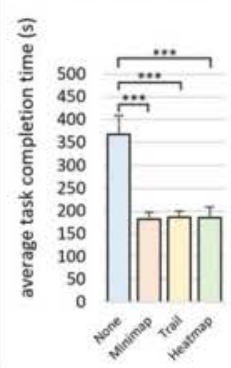

Figure 5: Average task completion times, i.e., the duration from the start to the end of a trial.

As depicted in Figure 5, task completion times differed significantly between groups for all three tasks: Maze Exploration $\left(F(3,44)=31.9, p<.001, \eta^{2}=.68\right)$, Naive Search $\left(F(3,44)=23.5, \quad p<.001, \eta^{2}=.62\right)$ and Informed Search $\left(F(3,44)=11.5, p<.001, \eta^{2}=.44\right)$. For all three tasks, Tukey-HSD post-hoc tests revealed that the condition withou any orientation-supporting tool (None) was significantly different from all others $(p<.05)$. Apart from that, only for the Naïve Search task completion times were significantly longer in the Minimap condition than both in the Heatmap and Trail condition.

\subsubsection{Task Accuracy}

Task accuracy was measured as the percentage of markers found in the exploration tasks (trials 1 and 4 ). We only assessed the accuracy for these two tasks because the number of markers in the other two tasks was predetermined, and the task could, therefore, only be completed with all markers found. In the maze exploration, four markers were distributed in the maze, whereas in the 'real-world' inspired exploration, ten markers (diamonds) were placed in the more realistic environment. In the first task, data were normally distributed and statistical tests indicate significant differences $(F(3,44)=5.3$, $p=.003, \eta^{2}=.27$ ). For the fourth task, the non-parametric KruskalWallis test reveals significant differences between used orientationsupporting aids $\left(H(3)=15.30, p=.002, \varepsilon^{2}=.33\right)$.

\section{Task Accuracy in Exploration Tasks}
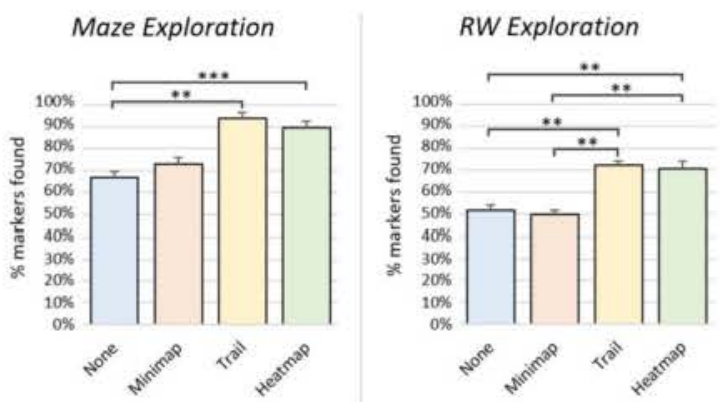

Figure 6: Mean values of task accuracy, measured as the percentage of markers found in the two exploration tasks (trial 1 and trial 4).
As shown in Figure 6, in the maze exploration task participants found significantly more markers in the Heatmap and Trail condition than in the None condition $(p<.05)$. The same applies to the real-world inspired exploration task, where in addition task accuracy was significantly lower in the Minimap condition compared to the Heatmap and Trail condition.

\subsubsection{Spatial Exploration}

We assessed the spatial exploration of participants in two ways On the one hand, we evaluated how much of the accessible space participants actually covered in the exploration tasks (trials 1 and 4), and on the other hand, how much space was redundantly visited in all tasks.

Explored area The percentage of space explored was calculated on the basis of participants' movement data. All accessible areas were divided into $1.5 \times 1.5 \mathrm{~m}$ tiles. Once a participant visited a tile, it was marked respectively. In the maze exploration task (trial 1) the $15 \times 15 \mathrm{~m}$ maze was divided into 100 tiles, whereas in the real-world inspired exploration task, the virtual compound of $1000 \mathrm{~m}^{2}$ was divided into 1000 tiles. Data in the maze exploration task were normally distributed and indicate significant differences $\left(F(3,44)=9.45, p<.001, \eta^{2}=.39\right)$. As shown in Figure 7 , in the None condition participants explored significantly less space of the maze compared to participants in the Heatmap or Trail condition $(p<.01)$. Additionally, there was a significant difference between the Trail and Minimap conditions. In the real-world inspired exploration task, data were non-normally distributed, and no significant differences between provided tools could be found $(H(3)=2.32, p=.509)$.

\section{Area Coverage in Exploration Tasks}

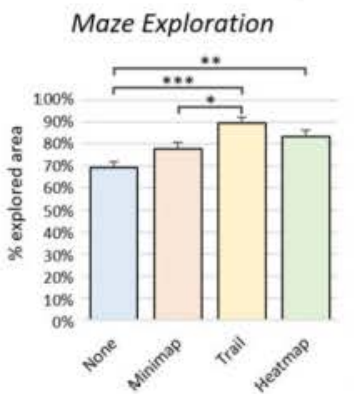

RW Exploration

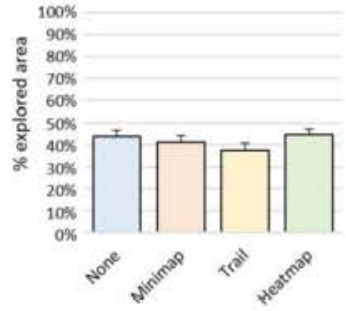

Figure 7: Mean values of exploration coverage of the accessible space in exploration tasks (trial 1 and 4).

Redundantly visited space To calculate the area that participants visited several times, we counted the tiles that registered the player more than once. Data were normally distributed only for the naïve search task and the real-world inspired exploration task. Statistical tests indicate significant differences for the naïve search task and the informed search task: Maze Exploration $(H(3)=3.08, p=$ $.380)$, Naïve Search $\left(H(3)=16.97, p=.001, \varepsilon^{2}=.36\right)$, Informed Search $\left(F(3,44)=6.16, p=.001, \eta^{2}=.30\right)$, Real-World Inspired Exploration $(F(3,44)=.917, p=.440)$.

As shown in Figure 8, in the naïve search task participants visited more areas redundantly in the None condition compared to all other conditions. In the informed search task this difference was only found between the None and Heatmap condition. However, in this task participants visited more space redundantly in the Minimap condition than in the Heatmap or Trail condition. 


\section{Redundant Visits in Search Tasks}
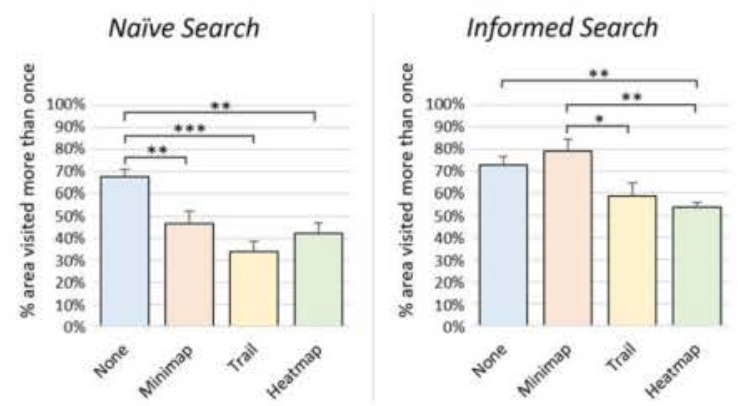

Figure 8: Mean percentage of areas that were visited more than once. Shown are tasks with significant differences: the naive search task (trial 2) and the informed search task (trial 3).

\subsubsection{Retrace Performance}

Participants' retrace skills were assessed in two ways. Firstly, in the naïve search and informed search tasks, as the time needed to return to the starting position after the task was completed. Secondly, only in the informed search task, as the time, distance, and error between two known markers. In this task, participants were asked to collect three markers in the right order. As participants always found the markers in the order 3,2, and then 1, they 'knew' the location of the second and third marker as soon as they had collected the first marker. Therefore, we could measure the variables as mentioned above for these portions. We assessed the time it took from one marker to the next, the distance participants covered between them, and the error, i.e., how often they tried to collect the third marker immediately after they had collected the first one.

Analyzing the time, that participants needed to return to the starting position after the last marker was found, showed no significant differences for the informed search task $(F(3,44)=1.76, p=.170)$ but for the naïve search task $\left(F(3,44)=3.6, p=.022, \eta^{2}=.11\right)$ In this task, participants needed significantly more time without an orientation-supporting tool (None) as if they were provided with the minimap (Minimap).

Considering only the informed search task, in which we assessed participants' ability to trace back their way to a previously found marker, for both retracings significant differences in time emerged: from the first to the second marker $\left(F(3,44)=3.1, p=.037, \eta^{2}=\right.$ $.17)$ and from the second to the third marker $(F(3,44)=4.1, p=$ $.012, \eta^{2}=.19$ ). Figure 9 depicts mean values of times participants needed to get from one marker to the next in the right order. In both cases the times in the None condition were significantly longer than in the Minimap condition $(p<.05)$. Furthermore, the time for tracing from the second to the third marker was longer in the None condition compared to the Trail condition. Similar results emerged when considering the variable distance. The analysis of the error rates did not reveal significant differences $(F(3,44)=0.55$, $p=.649$ ).

\subsubsection{Memorization}

After trial 2 and trial 3, participants were asked to choose the correct ground plan of the maze they previously had walked through from three options. Since the first two trials took place in the same maze, we asked participants to fill in the memorization questionnaire only once after the second, and once after the third trial. The memorization score is binary for each participant $(0=$ wrong choice, 1 = correct choice). Therefore, we computed Pearson's chi-squared test to detect significant associations between orientation-supporting

\section{Retrace Times in Informed Search Task}

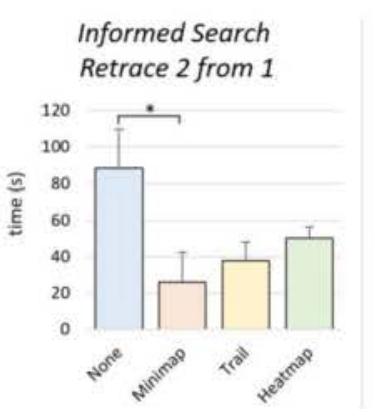

Informed Search

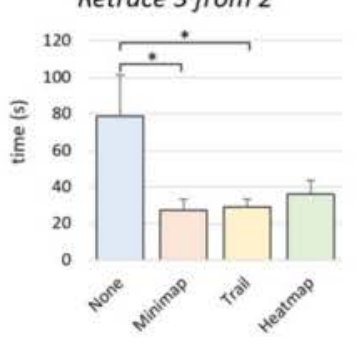

Figure 9: Mean values of retrace times, measured as the time it took participants to navigate from one marker to another in the informed search task (trial 3).

tools and answers to the memorization question. In the first memory assessment (after the naïve search task) there was a significant association between the two variables $\left(\chi^{2}(3)=27.45, p<.001\right.$, $\varphi=.57$ ). In the second memorization assessment (after the informed search task) we could not find any significant relationship $\left(\chi^{2}(3)=5.85, p=.054\right)$.
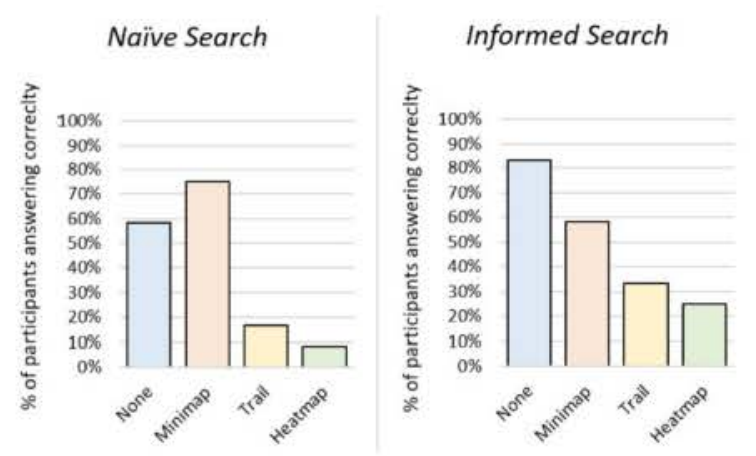

Figure 10: Memorability assessment. Percentage of participants choosing the correct ground plan in the memorability questionnaire after completing trial 2 and trial 3 .

As shown in Figure 10, in the first memory assessment, more participants were able to choose the right ground plan in the None $(n=7)$ and Minimap $(n=9)$ conditions than in the Trail $(n=2)$ and Heatmap $(n=1)$ conditions. In the second assessment, the number of participants who could recall the environment also differed: In the None condition ten participants chose the right ground plan, in the Minimap condition seven participants succeeded $(n=7)$ and in the other two conditions considerably fewer subjects gave the correct answer (Trail: $n=4$, Heatmap: $n=3$ ).

\subsubsection{Task Load \& Difficulty}

After each task, participants completed the NASA Task Load Index (TLX), assessing the subjective task load. Statistical analysis showed no significant differences between orientation-supporting tools for any task. In the final questionnaire participants rated the difficulty of the given task for each of the first three trials. In all cases data were normally distributed. Significant differences only emerged in the naïve search task (Maze Exploration: $F(3,44)=.86$, 
$p=.471 ;$ Naive Search: $F(3,44)=9.78, p<.001, \eta^{2}=.40 ;$ Informed Search: $F(3,44)=2.06, p=.120$ ). In the naïve search task, participants rated the task as more difficult when no supporting tool was available (None), compared to the conditions Trail and Minimap. Additionally, participants in the Heatmap condition found the task more difficult than in the Minimap condition.

\subsubsection{Subjective User Ratings}

In a final questionnaire, participants were asked to assess themselves. Among other things, they assessed their general ability to orient themselves and their ability to get an overview of the mazes they had explored. The questionnaire items were: (I) How good was your orientation in the first three maze scenarios? $(1=$ very bad to $5=$ very good) and (II) Was it easy/hard for you to keep the overview of the scenario? $(1=$ very hard to $5=$ very easy $)$.

Significant differences were found for both ratings: (I) $F(3,44)=$ $6.19, p=.001, \eta^{2}=.30$; (II) $F(3,44)=4.67, p=.006, \eta^{2}=.24$. Participants in the Minimap $(M=3.24)$ condition reported having better orientation than those in the None $(M=1.76)$ and Heatmap $(M=1.84)$ conditions. Moreover, participants in the Minimap $(M=$ 3.40) condition rated their ability to maintain an overview on average better than those in the None $(M=2.08)$ condition.

\subsubsection{Qualitative Results}

At the end of the study, a semi-structured interview was conducted to obtain qualitative feedback. We gathered, among others, their opinion on the helpfulness of the provided tool, strategies that were used to solve tasks, and feedback on how orientation could be improved

Most participants stated that the orientation-supporting tool provided helped them to solve the tasks $(n=34 ; 94 \%)$. As reasons for this, three participants stated that the tool (heatmap and trail) provided outstanding characteristics that could be used as orientational anchors in an environment where everything else looked very similar and monotonous. Two participants in the minimap condition stated that the aid helped them to keep track of the maze. One participan in the Trail condition stated that the overlapping trails were 'messy' and sometimes 'confusing'.

When asked whether they used a strategy, six participants elaborated on how they used a strategy that directly involved the orientation-supporting technique. Two used the heatmap, and one used the trail to create marks by intentionally moving in a certain pattern.

Participants were asked to estimate their performance in a realworld maze, compared to being in a virtual reality environment. Most $(n=39)$ assumed that they would have done better if they had been in a real rather than a virtual maze. The reasons they gave included the following: Teleportation hampers orientation $(n=11)$, presence of real-world artifacts $(n=6)$, movement is slower in the real world $(n=2)$, movement is easier $(n=2)$, better orientation in the real world $(n=2)$. Conversely, there were also some participants $(n=7)$ who estimated their performance to be worse in a real-world scenario due to faster physical exhaustion $(n=4)$ and the lack of teleportation for fast movement $(\mathrm{n}=2)$.

As suggestions for possible improvements in orientation, participants wished for a more diverse environment that provides more distinct anchors for orientation $(n=10)$. Moreover, some partici pants suggested increasing the room provided for physical walking $(n=5)$. Eight participants who were not in the Minimap condition even asked for a minimap as additional support.

\section{Discussion}

For all three tasks, participants were faster when orientationsupporting tools were provided, confirming a general benefit of orientation-supporting tools, which is consistent with previous research $[13,18,28,43]$. In exploration tasks, participants were more accurate and explored more space when provided with a Trail or a
Heatmap. In the naïve search task, participants visited less space redundantly when any aid was provided. Moreover, participants with a Minimap were faster in tracing back to previous locations. Participants' self-assessment regarding their subjective orientation and overview capabilities in general also revealed that providing a Minimap was advantageous in comparison to when no orientationsupporting tool was available. Hence, we can accept hypothesis $\mathrm{HI}$ as we could show that the provision of any of the three evaluated techniques improved participants' orientation.

In the maze exploration task, participants explored more space when a Trail or a Heatmap was provided compared to when no aid was given. As a matter of fact, users in the Trail condition outperformed those in the Minimap condition in that aspect. The two techniques Trail and Heatmap performed similarly in terms of accuracy. In the maze exploration task, participants found more markers when using one of the two techniques than users without aid. If we take a closer look at the real-world inspired exploration task, the two techniques also outperformed the Minimap. However, between the two techniques Trail and Heatmap no differences emerged. Therefore, we can only partially accept hypothesis $H 2$, since the Heatmap could only prevail against the None and Minimap condition in terms of exploration accuracy and efficiency.

Participants rated their subjective overview abilities as best when equipped with a Minimap, similar to results from a study by Kotlarek et al. [28] in which participants prefererred a 3D minimap over landmarks and waypoints. The memorability assessment revealed that participants with a Minimap could remember the ground plan of the maze they explored better than participants in the Trail condition. In the nailve search task, participants with the Minimap were faster compared to participants without an orientation-supporting tool or with one of the other two techniques. Therefore, we can partially accept hypothesis $\mathrm{H3}$, stating that the provision of a Minimap increases the overview of users and thus promotes orientation.

In the informed search task, participants were able to navigate from the second to the third marker faster when the Trail was provided than if no aid was available. This could be due to the visualization of the trail that marks the path taken earlier. However, it seems that traceability skills were even further enhanced with a Minimap. With a Minimap, participants could retrace from the first to the second and from the second to the third marker faster compared to the condition in which no aid was provided. This could be due to an increased overview in the Minimap condition. As soon as a marker was found, participants memorized its location and could return to it more easily with the help of the Minimap. No significant differences emerged between the techniques. Focusing on the time participants needed to return from the last found marker to the starting position, only in the naïve search task, it took significantly less time with the Minimap than without any help. These findings are in line with participants' subjective assessments of their orientation and overview-keeping abilities in the final questionnaire. Therefore, we have to reject hypothesis $H 4$, which is based on previous work by Ruddle et al. [43] and suggests that Trail is the most efficient technique for retracting tasks.

Interestingly, participants had less difficulty in remembering the maze correctly when no navigational tool was available compared to the Trail and Heatmap condition. It is possible that participants in these two conditions relied solely on the navigational aids to solve the task, which ultimately prevented participants from creating an inner image of the maze, making it more difficult for them to recall the ground plan later on. The Minimap, on the other hand, directly represented the ground plan of the maze, which made it relatively easy for participants to recall it afterward.

In the naïve search task, participants visited a larger area redundantly when no aid was provided. In the informed search task, only in the Heatmap condition. a significantly smaller area was visited redundantly by participants compared to the condition in which no 
orientation-supporting tool was available. Participants with a Minimap visited more areas redundantly compared to participants in the Trail or Heatmap condition. This could be an indication that the provision of a local overview of movement history as provided by the Trail and Heatmap techniques is more important for space-efficient, non-redundant navigation than a global overview of the environmen as provided by a Minimap. Similarly, the task completion time was higher when participants used the Minimap for the naïve search task compared to participants using Trail or Heatmap. This could be due to changed user behaviour when the overview of the entire environment is given and the amount of hidden markers is known. Participants probably tried to quickly navigate through the maze to find all three markers, rather than carefully inspecting each corner as in the exploration tasks, which may have led them to frequently circle back to areas they were close by earlier.

The Minimap performed worse in terms of user accuracy in the real-world inspired scenario compared to the maze exploration task In the real-world inspired task, participants in the Trail or Heatmap conditions collected significantly more markers. This could be due to the limited scalability of the minimap approach. With an increasing area of the environment, the minimap is zoomed out in order to fit the entire environment on the handheld map. As a result, everything depicted becomes smaller and harder to read.

In sum, results suggest that the deployment of orientationsupporting tools can be beneficial in exploratory search and wayfinding tasks. Depending on the task, different orientation-supporting tools are recommendable. In case retracing is a central element of the task at hand, our results suggest the use of a Minimap. In order to avoid redundant visits of the same location, the direct visualization of visited places by means of Heatmap and Trail visualizations is recommended. With the Trail visualization, participants were able to cover more of the available area on average, leaving less space unexplored. One reason for this could be that the trail enabled participants to encode the history of their movements. While the Heatmap representation solely highlights areas in which the user was previously located, the Trail visualization also provides the context of how the respective location was visited, i.e., from where and to where. Based on our results, we propose that future systems should consider the provision of visual aids in any scenarios involv ing search and exploration tasks, as it can reduce disorientation and task completion times while increasing accuracy. Furthermore, our results underline that less is sometimes more, as we found distracting factors of visualization elements on memorability. Hence, when memorability is an important aspect in a given task, one should consider refraining from constantly showing visual cues that are embedded in the environment (e.g., heatmaps or trails), but rather enable switching them on and off or deploying visual aids that are detached from the environment (e.g., minimaps).

\section{LIMITATIONS \& FUTURE WORK}

Some limitations need to be taken into account. The results of the current study only allow statements about the performance of the three selected orientation-supporting tools in a planar environment where a user walks on a plane underground. Results could be different if a three-dimensional space is to be explored, for instance. if the task is to explore an abstract 3D scatterplot. In such cases Heatmap and Minimap may not be applicable or only in a limited form. Furthermore, we used a relatively small environment and a short search task for our study. In case the environment is much larger, different levels of scalability are to be expected from the three tools evaluated in this study. Ruddle et al. [43], for example, found that overlapping trails are a major limitation of the Trail technique in long exploration tasks. To overcome this, the basic form could be extended by color-coding the time on the trail, reducing the size of the trail depending on the time or even blinding out old segments of the trail. Also, for the Minimap challenges become apparent when the environment is much larger. In this case, it may no longer be possible to display the entire ground plan on the minimap, but only a small section of it.

In the present research, we were interested in the performance of different orientation-supporting tools when the primary movement mode is teleportation. We strive to more precisely look into the impact of teleportation in future work by comparing the performance of different orientation-supporting tools when other movement options are offered. The performance of tools could change if participants could walk naturally in the $15 \times 15 \mathrm{~m}$ space or use slow transitions between the starting point and the selected teleport destination.

The evaluation of different orientation-supporting tools was specifically tailored to their use in an abstract virtual reality environment. Nonetheless, all deployed techniques can also be used in augmented reality applications. However, we assume that the type of movement used - i.e., teleportation - had a substantial influence on the performance of the techniques and could, therefore, be a topic for future research. Findings, for example, about retrace and overview capabilities of the individual techniques, could be used as a starting point for subsequent research in AR. Similarly, it would be interesting to assess the actual impact of the realisticness of an environment on orientation. In the present study, we mainly used abstract mazes that could not only have led to stronger effects as desired, but also to other effects, which would make it difficult to transfer knowledge gained from the experiment with abstract mazes to everyday use cases.

Future research should also address combinations of the presented techniques, exploring if and how trade-offs could be balanced out. For example, the Heatmap and Minimap techniques could be merged into a single one combining the advantages of both techniques. This way, the user could keep an overview and, at the same time, be aware of unexplored locations.

\section{ConClusions}

The present study investigated three orientation-supporting tools for various exploration and search tasks in virtual reality environments. We provided the common technique of teleportation for spatial movement and used mazes as virtual environments to promote a loss of orientation. Results indicate that the tools supported users in exploration and naïve search tasks. However, we found that providing Trails and Heatmaps had a negative effect on participants memorization ability in informed search tasks. The provision of a Minimap seemed to improve users' retrace ability more than the Trail technique, which was unexpected as the Trail depicts the history of a user's movement directly in the visualization. Generally, our study suggests a benefit of orientation-supporting tools and shows the strengths and weaknesses of different approaches for different tasks.

\section{ACKNOWLEDGMENTS}

This work was funded by the Deutsche Forschungsgemeinschaft (DFG, German Research Foundation) within the projects $\mathrm{A} 03$ and A08 of TRR 161 (Project-ID 251654672).

\section{REFERENCES}

[I] M. Allahyar and E. Hunt. The assessment of spatial orientation using virtual reality techniques. International Journal of Testing, 3(3):263 275, 2003, doi: $10.1207 /$ S15327574UT0303.5

[2] M. Beetz, N. von Hoyningen-Huene, B. Kirchlechner, S. Gedikli, F. Siles, M. Durus, and M. Lames. Aspogamo: Automated sports game analysis models. International Journal of Computer Science in Sport, 8(1):1-21, 2009. doi: 10.1.1.184.9785

[3] J. Bhandari, P. R. MacNeilage, and E. Folmer. Teleportation without spatial disorientation using optical flow cues. In Graphics interface. pp. 162-167, 2018. doi: 10.20380/GI2018.22 
[4] D. A. Bowman, E. T. Davis, L. F. Hodges, and A. N. Badre. Maintain ing spatial orientation during travel in an immersive virtual environment. Presence, 8(6):618-631, 1999, doi: 10.1162/105474699566521

[5] S. Burigat and L. Chittaro. Navigation in $3 \mathrm{~d}$ virtual environments: Effects of user experience and location-pointing navigation aids. International Journal of Human-Computer Studies, 65(11):945-958, 2007 doi: 10.1016/j.ijhcs.2007.07.003

[6] T. Büring, J. Gerken, and H. Reiterer. Usability of overview-supported zooming on small screens with regard to individual differences in spatial ability. In Proceedings of the working conference on Advanced visual interfaces, pp. 233-240, 2006, doi: 10.1145/1133265.1133310

[7] L. Chittaro and S. Burigat. 3d location-pointing as a navigation aid in virtual environments. In Proceedings of the working conference on Advanced visual interfaces, pp. 267-274, 2004. doi: 10.1145/989863. 989910.

[8] D. Cliburn, T. Winlock, S. Rilea, and M. Van Donsel. Dynamic landmark placement as a navigation aid in virtual worlds. In Proceedings of the 2007 ACM symposium on Virtual reality software and technology, pp. 211-214. ACM, 2007. doi: 10.1145/1315184.1315225

[9] E. Coluccia and G. Louse. Gender differences in spatial orientation: A review. Journal of environmental psychology, 24(3):329-340, 2004. doi: $10.1016 /$ j.jenvp.2004.08.006

[10] R. Darken and B. Peterson. Spatial orientation, wayfinding, and representation. Handbk Virtual Environ, 2002, 11 2001. doi: 10.1201/ b17360-24

[11] R. P. Darken and W. P. Banker. Navigating in natural environments: A virtual environment training transfer study. In Proceedings. IEEE 1998 Virtual Reality Annual International Symposium (Cat. No. 98CB36180), pp. 12-19. IEEE, IEEE, 1998, doi: 10.1109/VRAIS.1998.658417

12] R. P. Darken and H. Cevik. Map usage in virtual environments: Orientation issues. In Proceedings IEEE virtual reality (cat, no. 99CB36316), pp. 133-140. IEEE, IEEE, 1999. doi: 10.1109/VR. 1999.756944

[13] R. P. Darken and J. L. Sibert. A toolset for navigation in virtual environments. In ACM symposium on User interface software and technology, pp. 157-165. ACM, 1993. doi: 10.1145/168642.168658

[14] R. P. Darken and J. L. Sibert. Wayfinding strategies and behavion in large virtual worlds. In Proceedings of the SIGCHI conference on Human factors in computing systems, pp. 142-149. ACM, 1996. doi: $10.1145 / 238386.238459$

15] C. Donalek, S. G. Djorgovski, A. Cioc, A. Wang, J. Zhang, E. Lawler, S. Yeh, A. Mahabal, M. Graham, A. Drake, et al. Immersive and collaborative data visualization using virtual reality platforms. In 2014 IEEE International Conference on Big Data (Big Data), pp. 609-614 IEEE, 2014. doi: 10.1109/BigData.2014.7004282

[16] D. Fisher. Hotmap: Looking at geographic attention. IEEE transactions on visualization and computer graphics, 13(6):1184-1191, 2007. doi: 10.1109/TVCG.2007.70561

[17] C. Freksa. Spatial cognition an ai perspective. In Proceedings of the 16th European Conference on Artificial Intelligence, pp. 1122-1128. IOS, Press, 2004. doi: 10.5555/3000001.3000289

[18] D. Grammenos, M. Filou, P. Papadakos, and C. Stephanidis. Virtual prints: Leaving trails in virtual environments. In EGVE, vol. 2, pp. 131-138. The Eurographics Association, 2002. doi: 10.2312/EGVE/ EGVE02/131-138

[19] S. G. Gustafson and P. P. Irani. Comparing visualizations for tracking off-screen moving targets. In $\mathrm{CHI}^{\prime} 07$ Extended Abstracts on Human Factors in Computing Systems, pp. 2399-2404, 2007. doi: 10.1145/ 1240866.1241014

[20] R. A. Haddad, A. N. Akansu, et al. A class of fast gaussian binomia filters for speech and image processing. IEEE Transactions on Signal Processing, 39(3):723-727, 1991. doi: 10.1109/78.80892

[21] S. G. Hart and L. E. Staveland. Development of nasa-tlx (task load index): Results of empirical and theoretical research. In Advances in psychology, vol. 52, pp. 139-183. Elsevier, Amsterdam, Netherlands, 1988. doi: $10.1016 /$ S0166-4115(08)62386-9

[22] M. Hegarty and D. Waller. A dissociation between mental rotation and perspective-taking spatial abilities. Intelligence, 32(2):175-191, 2004 doi: $10.1016 / \mathrm{j}$.intell.2003.12.001

[23] L. J. Hettinger and G. E. Riccio. Visually induced motion sickness in virtual environments. Presence: Teleoperators \& Virtual Environments,
1(3):306-310, 1992, doi: 10.1162/pres. 1992.1.3.306

[24] HTC Vive developer website. https://www.vive.com/eu/, 2020. Accessed: 2020-08-01.

[25] W. Jentner, M. Kraus, N. Weiler, T. Kilian, and D. A. Keim. Visua analytics system for semi-automatic $4 \mathrm{~d}$ crime scene reconstruction. In 4th International Symposium on Big Data Visual and Immersive Analytics, 2018.

[26] R. S. Kennedy, N. E. Lane, K. S. Berbaum, and M. G. Lilienthal Simulator sickness questionnaire: An enhanced method for quantifying simulator sickness. The international journal of aviation psychology, 3(3):203-220, 1993. doi: 10.1207/s 15327108 ijap0303.3

[27] S. U. König, A. Keshava, V. Clay, K. Ritterhofer, N. Kuske, and P. König. Embodied spatial knowledge acquisition in immersive virtual reality: Comparison of direct experience and map exploration. bioRxiv. 2020, doi: $10.1101 / 2020.01 .12 .903096$

[28] J. Kotlarek, I.-C. Lin, and K.-L. Ma. Improving spatial orientation in immersive environments. In Proceedings of the Symposium on Spatial User Interaction, pp. 79-88, 2018, doi: 10.1145/3267782.3267792

[29] D. Kutsenko. Unity 3D Asset industrial scenario map. https://assetstore.unity.com/packages/3d/environments /industrial/rpg-fps-/game-assets-for-pc-mobile-indust rial-set-v2-0-86679,2020. Accessed: 2020-08-01.

[30] E. Langbehn, P. Lubos, and F. Steinicke. Evaluation of locomotion techniques for room-scale vr: Joystick, teleportation, and redirected walking. In Proceedings of the Virtual Reality International ConferenceLaval Virtual, pp. 1-9, 2018. doi: 10.1145/3234253.3234291

[31] K. Lynch. The image of the city, vol. 11. MIT press, 1960. doi: 10. $1177 / 000271626133600164$

[32] M. Macik. Cognitive aspects of spatial orientation. Acta Polytechnice Hungarica, 15(5), 2018. doi: 10.12700/aph. 15.5.2018.5.9

[33] E. A. Maguire, N. Burgess, and J. OKeefe. Human spatial navigation: cognitive maps, sexual dimorphism, and neural substrates. Curremt opinion in neurobiology, 9(2):171-177, 1999. doi: 10.1016/\$0959 4388(99)80023-3

[34] S. D. Moffat, A. B. Zonderman, and S. M. Resnick. Age differences in spatial memory in a virtual environment navigation task. Neurobiology of aging, 22(5):787-796, 2001. doi: 10.1016/S0197-4580(01)00251-2

[35] J. Müller, S. Butscher, S. P. Feyer, and H. Reiterer. Studying collaborative object positioning in distributed augmented realities. In Proceed ings of the 16th International Conference on Mobile and Ubiquitous Multimedia, pp. 123-132. ACM Press, 2017. doi: 10.1145/3152832. 3152856

[36] H.-S. Park, J. W. Yoon, J. Kim, K. Iseki, and M. Hallett. Development of a vr-based treadmill control interface for gait assessment of patients with parkinson's disease. In 2011 IEEE International Conference on Rehabilitation Robotics, pp. 1-5. IEEE, 2011. doi: 10.1109/ICORR. 2011.5975463

[37] R. Passini. Wayfinding in architecture. Environmental Design Series (Stroudsburg, Pa.), V. 4. Van Nostrand Reinhold, 1984

[38] C. Perin, R. Vuillemot, and J.-D. Fekete. Soccerstories: A kick-off for visual soccer analysis. IEEE transactions on visualization and computer graphics, 19(12):2506-2515, 2013. doi: 10.1109/TVCG. 2013.192

[39] M. Peters, B. Laeng, K. Latham, M. Jackson, R. Zaiyouna, and C. Richardson. A redrawn vandenberg and kuse mental rotations test-different versions and factors that affect performance. Brain and cognition, 28(1):39-58, 1995. doi: 10.1006/brcg. 1995.1032

[40] M. E. Portman, A. Natapov, and D. Fisher-Gewirtzman. To go where no man has gone before: Virtual reality in architecture, landscape architecture and environmental planning. Computers, Environment and Urban Systems, 54:376-384, 2015. doi: 10.1016/j.compenvurbsys .2015 .05 .001

[41] S. Razzaque, Z. Kohn, and M. C. Whitton. Redirected Walking. In Eurographics 2001 - Short Presentations. Eurographics Association, 2001. doi: 10.2312 egs. 20011036

[42] B. E. Riecke and J. Schulte-Pelkum. Perceptual and cognitive factors for self-motion simulation in virtual environments: how can selfmotion illusions (vection) be utilized? In Human walking in virtual environments, pp. 27-54. Springer, 2013, doi: 10.1007/978-1-4419 $-8432-6.2$ 
[43] R. A. Ruddle. The effect of trails on first-time and subsequent navigation in a virtual environment. In IEEE Proceedings. VR 2005. Virtual Reality, 2005., pp. 115-122. IEEE, IEEE, 2005. doi: 10.1109/VR.2005 .1492761

[44] M. Sarkar and M. H. Brown. Graphical fisheye views of graphs. In Proceedings of the SIGCHI conference on Human factors in computing systems, pp. 83-91, 1992. doi: 10.1145/142750.142763

[45] L. H. Sloot, M. M. Van der Krogt, and J. Harlaar. Effects of adding a virtual reality environment to different modes of treadmill walking. Gait \& posture, 39(3):939-945, 2014. doi: 10.1016/j.gaitpost.2013.12. 005

[46] S. D. Steck and H. A. Mallot. The role of global and local landmarks in virtual environment navigation. Presence: Teleoperators \& Virtual Environments, 9(1):69-83, 2000. doi: 10.1162/105474600566628

[47] S. Stellmach, L. Nacke, and R. Dachselt. Advanced gaze visualization for three-dimensional virtual environments. In Proceedings of the 2010 symposium on eye-tracking research \& Applications, pp. 109-112. ACM, 2010. doi: 10.1145/1743666.1743693

[48] Unity 3D developer website. https://www.unity3d.com/, 2020. Accessed: 2020-08-01.

[49] H. A. Van Veen, H. K. Distler, S. J. Braun, and H. H. Bülthoff. Navigating through a virtual city: Using virtual reality technology to study human action and perception. Future Generation Computer Systems, 14(3-4):231-242, 1998. doi: 10.1016/S0167-739X(98)00027-2

[50] B. Williams, G. Narasimham, B. Rump, T. P. McNamara, T. H. Carr, J. Rieser, and B. Bodenheimer. Exploring large virtual environments with an hmd when physical space is limited. In Proceedings of the 4th symposium on Applied perception in graphics and visualization. $\mathrm{pp}$. 41-48. ACM, 2007. doi: 10.1145/1272582.1272590

[51] G. Zhou, Z. Tan, P. Cheng, and W. Chen. Modeling and visualizing $3 \mathrm{~d}$ urban environment via internet for urban planning and monitoring. In Proceeding XXth ISPRS Congress, in Istanbul, Turkey, pp. 12-23, 2004. doi: 10.1.1.131.1248 